\title{
Performance analysis of massive multiple input multiple output for high speed railway
}

\author{
Maharshi K. Bhatt ${ }^{1}$, Bhavin S. Sedani ${ }^{2}$, Komal Borisagar ${ }^{3}$ \\ ${ }^{1}$ Electronics and Communication Engineering Department, Government Engineering College, Bhuj, Gujarat, India \\ ${ }^{1,3}$ Gujarat Technological University, Ahmedabad, Gujarat, India \\ ${ }^{2}$ Electronics and Communication Engineering Department, L. D. College of Engineering, Ahmedabad, Gujarat, India
}

\begin{tabular}{l} 
Article Info \\
\hline Article history: \\
Received Dec 31, 2020 \\
Revised May 25, 2021 \\
Accepted Jun 8, 2021 \\
\hline
\end{tabular}

\section{Keywords:}

Carrier frequency offset Channel estimation error Doppler frequency offset Handover HSR Inter carrier interference Massive MIMO

\begin{abstract}
This paper analytically reviews the performance of massive multiple input multiple output (MIMO) system for communication in highly mobility scenarios like high speed Railways. As popularity of high speed train increasing day by day, high data rate wireless communication system for high speed train is extremely required. $5 \mathrm{G}$ wireless communication systems must be designed to meet the requirement of high speed broadband services at speed of around $500 \mathrm{~km} / \mathrm{h}$, which is the expected speed achievable by HSR systems, at a data rate of $180 \mathrm{Mbps}$ or higher. Significant challenges of high mobility communications are fast time-varying fading, channel estimation errors, doppler diversity, carrier frequency offset, inter carrier interference, high penetration loss and fast and frequent handovers. Therefore, crucial requirement to design high mobility communication channel models or systems prevails. Recently, massive MIMO techniques have been proposed to significantly improve the performance of wireless networks for upcoming $5 \mathrm{G}$ technology. Massive MIMO provide high throughput and high energy efficiency in wireless communication channel. In this paper, key findings, challenges and requirements to provide high speed wireless communication onboard the high speed train is pointed out after thorough literature review. In last, future research scope to bridge the research gap by designing efficient channel model by using massive MIMO and other optimization method is mentioned.
\end{abstract}

This is an open access article under the CC BY-SA license.

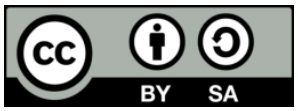

\section{Corresponding Author:}

Maharshi K. Bhatt

Electronics and Communication Engineering Department

Government Engineering College

Bhuj, PIN code- 370001, Gujarat, India

Email: maharshi88@gmail.com

\section{INTRODUCTION}

In massive multiple input multiple output (MIMO) system, a base station mounted more than hundred antennas provide simultaneous coverage to multiple users. Massive MIMO systems are nowadays emerged as newly designed cellular network architecture with numerous interesting features like achievable capacity can be increased by merely mounting additional antennas to currently active cell sites, a greater number of antennas significantly reduce uplink and downlink transmit power. Energy conservation is extremely necessary in existing scenario of the world and as per survey done in 2013, 69 Giga Watts power consumed world-wide telecommunication networks i.e. equivalent to power consumed by 12 New York cities in a year [1]. So massive MIMO is the prominent candidate that can dramatically reduce future power consumption of wireless telecommunication technology and achieve huge energy efficiency. This higher 
energy efficiency characteristic is not only relevant from an industrial benefit point of view but also environmental as well as safety of health concern related to wireless communications. Another feature is channel reciprocity, in which the overhead associated to channel training scales linearly with user terminals $(K)$ and is independent of the number of base station antennas $(N)$, if $N>K$, we can use the simplest linear detectors at receiver. Due to large number of base station antennas, effect of thermal noise, interference, and channel estimation errors vanish. When the number of BS antennas increases, the random channel vectors between the users terminals and the base station become pair wise orthogonal. Another major advantage of massive MIMO systems is that they facilitate us to reduce the transmitted power. On the uplink channel, reducing the transmit power of the user terminals will decrease rate of drain their batteries. The design and analysis of massive MIMO systems is a quite a new concept that is attracting extensive interest.

In this review paper, we have presented performance analysis of massive MIMO system for highly mobility scenario like high speed railways (HSR). High speed railway (HSR) is established in many countries and regions as one of the economic, secure, comfortable and punctual transportation system for medium to long distance travel. To provide highly user friendly environment and operational safety in the train, broadband services such as onboard high definition video surveillance, passenger high speed internet access, multimedia functionalities of train, railway emergency communications, online Television and IoT applications of railway, are nowadays very essential [2], [3]. In addition, record of maximum moving speed of high speed train is refreshing quickly. Some new challenges are raised by increasing the speed of train like inter carrier interference, carrier frequency offset, frequent and fast handover, high Doppler spread etc. "Field tests carried out in various countries, which show that existing $4 \mathrm{G}$ technology can only provide maximum of $4 \mathrm{Mbps}$ data rate to high speed trains" [4]. Expectations of future high speed trains are to provide services to passengers at a data rate of $150 \mathrm{Mbps}$ or more than that. Therefore, it is essential to design new technologies that meet the need of flawless communication technology in high speed train. Significant challenges of high speed railway communications are high channel fading, high penetration loss, inter carrier interference, time varying nature of channel, channel estimation errors, doppler frequency offset (DFO), carrier frequency offset and frequent handovers. In high mobility scenarios, severe channel estimation error occurs, which causes serious degradation in system performance [5], [6]. Doppler shift generates carrier frequency offset (CFO) which occurs because of difference between the frequency of the transmitter and receiver. Penetration loss in HSR systems is introduced by the metal sealed train coaches. Inter carrier interference (ICI) is introduced by doubly selective fading in high mobility systems in which channel changes inside single OFDM symbol and thus ruin the orthogonality inside the subcarriers.

Some newly emerged technologies are introduced to solve the above problems of future HSR communication systems such as $5 \mathrm{G}$ on $\mathrm{HSR}$, and $5 \mathrm{G}$ for railway (5G-R) have attracted much attention. These technologies are developed on the backbone of revolutionary transmission technologies like massive multiple MIMO, mobile relay, coordinated multipoint and millimeter-wave (mmWave) technology [7], [8]. To minimize penetration losses of propagation signals, mobile relay station (MRS) are mounted on roof of the train coaches. MRS provides multihop coverage from base stations to train travelers and thus signal quality improves. Massive MIMO technology improves channel capacity and hence increases data rate by installation of large number of antennas at base station hence it is extremely significant role player for improvement of performance of communication network. Throughput of wireless channel can be increased by Position added channel estimation scheme where portion of transmitter antenna transmits pilot symbols to estimate channel and hence no need to measure channel at the receiver by this method information bits can be increased by minimizing channel estimation bits at receiver [9]-[11]. Beamforming technique is used at antenna for high array gain. Other advantages of massive MIMO technology are high diversity gain and multiplexing gain which results in high power gain. But in highly mobile scenario, massive MIMO architecture requires huge number of transmitter antennas and hence dimension of antenna is increased and complexity of structure also increased. To evaluate performance of massive MIMO, designing network and testing in real field, a perfect channel model development is extremely essential [12].

First network is between backbone network which can be high speed $5 \mathrm{G}$ internet network and base station, the second network is between mobile relay station (MRS) mounted on roof of train and base station, and the last one is the MRS on the top of coach of a train coaches and travelers inside the train who are users. For such communications network, some promising communication technologies, such millimeter Wave, tera hertz (THZ) and massive MIMO using beamforming can be appropriate [13]. Massive MIMO will be introduced to provide high gains to overcome high path loss of mmWave and $\mathrm{THz}$ communications. On the other hand, co-ordinate multipoint technique can also be used in the combined cell to achieve high gain and simultaneously improve spectral efficiency in high speed railway communication system [14], [15]. In the combined network of coordinate multipoint technique, two or more base stations are connected to form a coordinated cell to exchange data. The network architecture of described HST communication system is illustrated in Figure 1. 




Figure 1. High speed railway communication architecture

\section{HSR CHANNEL MODEL}

Trains may experience different types of environmental conditions or scenarios while travelling. These scenarios can be approximately categorised into six scenarios of train passes from like open space, viaduct, hilly terrain, cutting, tunnels and stations. Moreover, wireless signal propagation can be categorised into five categories that is, inter train, train to infrastructure, infrastructure to infrastructure, intra train, inside the train station, and [16]. Table 1 shows various HSR channel models.

Table 1. Recent developments of HST channel models

\begin{tabular}{cccc}
\hline Name of Channel model & Frequency band & Deterministic /stochastic & Train speed $(\mathrm{km} / \mathrm{h})$ \\
\hline Ray tracing model & Mm Wave & Deterministic & 300 \\
GBSM & Mm Wave & Stochastic & 500 \\
QuaDRiGa-based channel model & Mm Wave & Stochastic & 500 \\
Dynamic channel model & Sub-6 GHz & Stochastic & 300 \\
FSMC & Sub-6 GHz & Stochastic & 350 \\
Propagation graph model & Sub-6 GHz & Stochastic & 198 \\
\hline
\end{tabular}

The Ray tracing channel model is deterministic channel model and operates in mmWave frequency band. It can provide accurate characterization of a propagation channel at a train speed of around $300 \mathrm{~km} / \mathrm{h}$ [16], [17]. One Ray is transmitted which measures information like delay, angle information and amplitude which is tracked and at receiver summation of all such rays gives information of channel for modelling.

The geometry-based stochastic channel models (GBSMs) is stochastic model and operate at mm Wave frequency band which can be classified into regular shaped GBSM and irregular shaped GBSM. Channel information can be derived from geometric relationship. The GBSM channel model is widely used in high speed railway channel modelling. QuaDRiGa-based channel model can support the mmWave band and applicable to train speed of $500 \mathrm{~km} / \mathrm{h}$. This model works based on scattering multipath and nonstationary of channel. The finite-state Markov model (FSMC) is stochastic model and applicable to train speed of $350 \mathrm{~km} / \mathrm{h}$. Propagation Graph Model is based on graph theory, and applicable for train speed of around $198 \mathrm{~km} / \mathrm{h}$.

\section{ANALYSIS OF MASSIVE MIMO TO ADDRESS CHALLENGES OF FUTURE HSRS}

Inter beam interference caused by high mobility scenario is discussed. Where massive MIMO technology which is one of the main key role player for the upcoming HSR system is introduced. Beamforming technology which is used at transmitter antenna in 5G technology and significantly reduce inter beam interference [18], [19]. In massive MIMO transmitter antenna transmits highly directive pencil narrow beams towards the receiver antenna of user, but unfortunately narrowing beam can reduce the probability of effective beamforming. Therefore, it is crucial to choose the appropriate beamwidth to transmit 
signal. Proposed technique uses optimal position-aware beamwidth adjustment strategy for high speed train scenario. An optimal beamwidth expression having good quality of service is derived by maximize the beam directivity.

Due to dominant line of sight signal, spatial temporal correlation exists, which is the characteristic of the propagation channels is discussed. Because of high channel correlation multiple antenna gain can't be achieved. However, it is necessary to control inter carrier interference (ICI) to decrease the strong channel correlation. In spatial modulation technique only one transmit antenna is activated and transmits information from the arrays of antennas. In spatial modulation, ICI is avoided which decreases channel correlation. Analysis of performance of spatial temporal correlated over spatial modulated massive MIMO for Rician fading channel and HSR scenario is conducted. Simulation result in [20] shows that, massive spatial modulated MIMO outperforms VBLAST except as shown in plot of Figure 2. Where $\mathrm{K}$ is Ricean factor, $\Delta_{\mathrm{t}}$ is normalized transmit antenna separation.

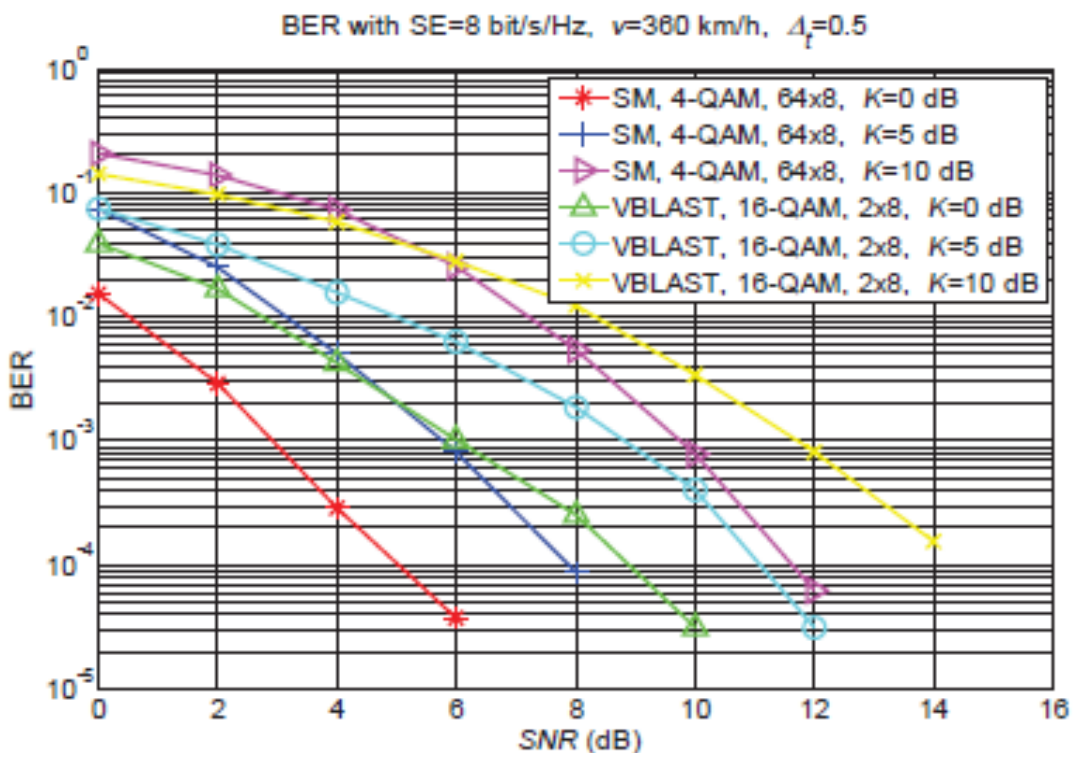

Figure 2. SNR vs. BER for velocity $=360 \mathrm{~km} / \mathrm{h}$ and spectral efficiency $8 \mathrm{bits} / \mathrm{s} / \mathrm{Hz}$

Uplink channel from high speed train to base station is analysed based on exact channel power spectrum density (PSD). Alsovia beamforming network optimization, further reduction in the channel time variation is proposed. Uniform linear array is installed on the roof of train which separates multiple doppler shifts in angle domain by beamforming technique. Channel time variation can be reduced by this method. In this each branch of beamforming contains compensated Doppler shift at Tx antenna. Channel power spectrum density (PSD) can be articulated as the product of a beam distortion function and pattern function. Beamforming network is optimized by common configurable amplitudes and phases (CCAP) parameter [21]. In this way, channel time variation and doppler shift can be reduced. As shown in Figure 3 Doppler spread is decreasing with increasing number of antennas $\mathrm{M}$ and also compared to MF beam formers, Doppler spread is reduced in optimized CCAP beamformers.

Angle domain doppler compensation can be applied for massive MIMO uplink channel for highly mobile environment. The time varying small scale fading channel is considered. Due to very high speed of train, multiple doppler frequency offsets (DFOs) are generated. By deploying large number of uniform linear array at transmitter antenna (mounted on the roof of train) a beamforming network having multiple parallel beamforming branches is developed, in which each transmitted signal pointed to one particular angle. Because of this structure, uplink signal of each branch will undergo only one dominant doppler frequency offset. Compensation of this single dominant DFO can be achieved at transmitter. Channel time variation can be efficiently reduced if large number of transmitter antennas are placed. Doppler spread is proportional to doppler frequency offset and reduces as $1 / \sqrt{ } M$ where $M$ is the number of transmit [22] as shown in Figure 4, where $\mathrm{f}_{\mathrm{d}} \mathrm{T}_{\mathrm{b}}$ is normalized maximum DFO.

Inter carrier interference (ICI) is introduced in high speed train because of fast time varying channel due to very high velocity of train. AWGN and Ricean fading downlink channels are focused to develop ICI reduction methods. "With the data of relative areas and speeds between comparing antenna pairs, ICI 
matrices in AWGN and Ricean fading channels are determinant, and they are having unity value" [23], [24]. Finally, two analogous low complexity ICI reduction techniques for fast time shifting nature are described, which analyse at the $300 \mathrm{~km} / \mathrm{h}$ velocity with same QoS that obtained in the case without ICI.

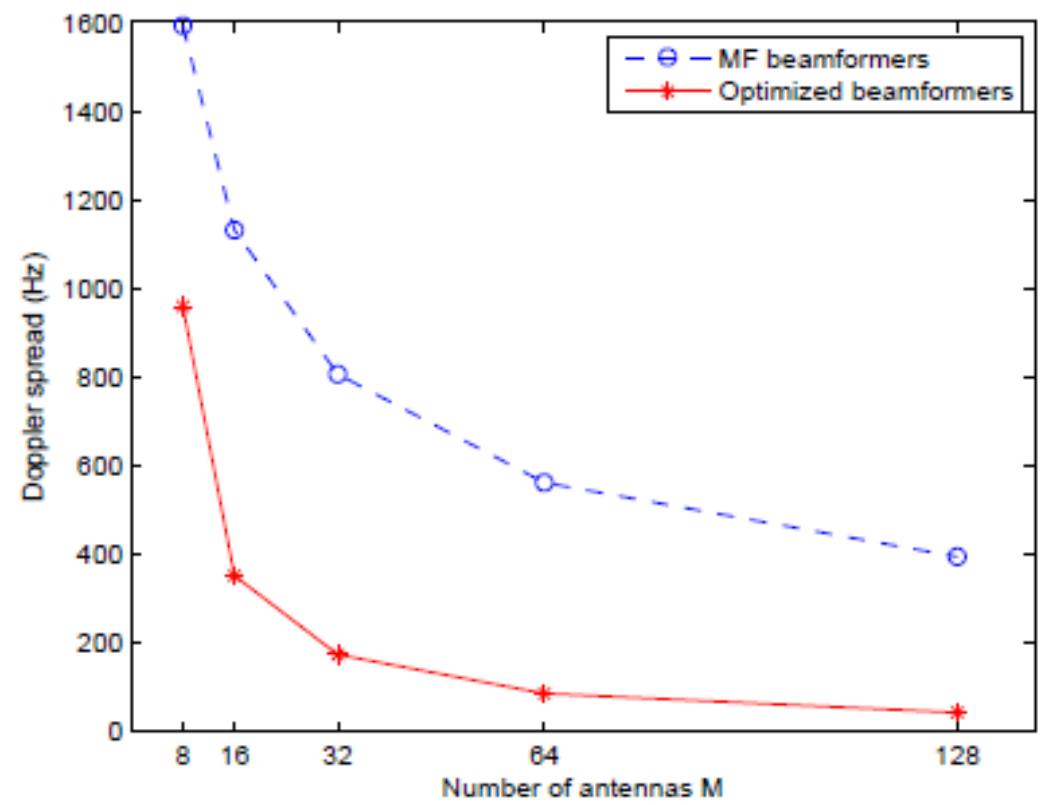

Figure 3. Comparison of Doppler spread computed with MF beam former and optimized CCAP beam former

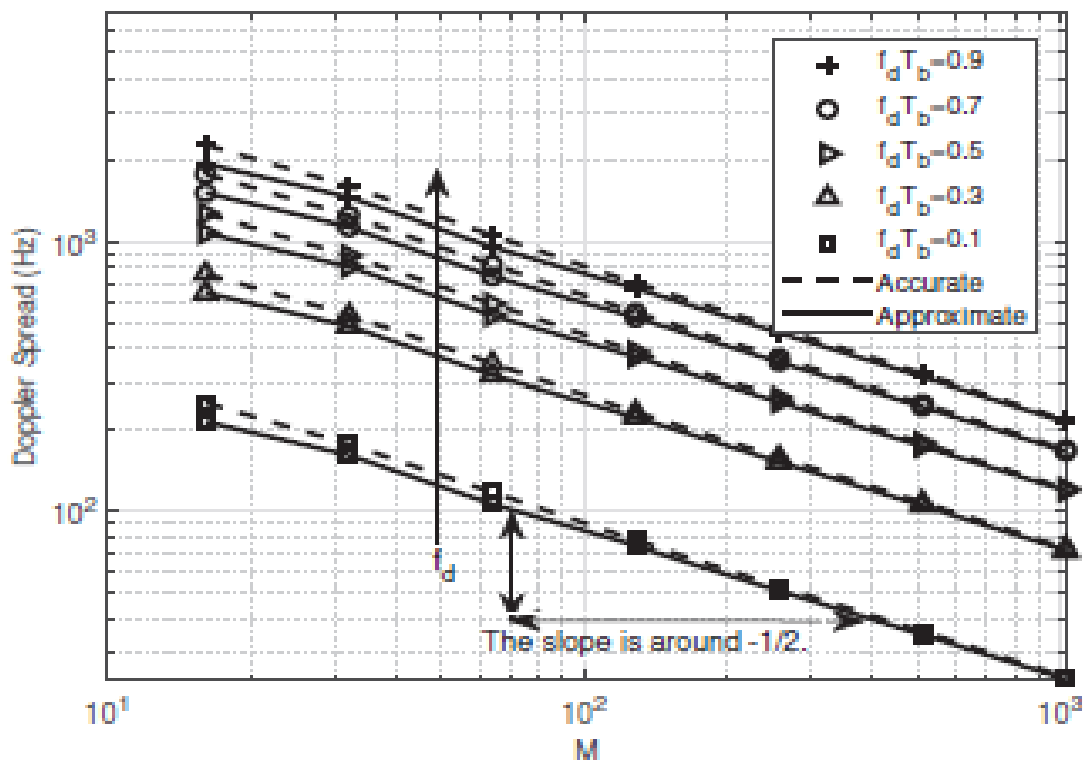

Figure 4. Doppler spread of uplink channel vs. Number of Tx antennas

Location based beamforming having less complex configuration for the massive MIMO system without considering the effect of inter beam interference, a unique solution can be achieved to increase the total system channel capacity in bits/Hz of base station is presented in high mobility scenario [25]. As shown in Figure 5, mobile service in bits/ $\mathrm{Hz}$ is higher in resource allocation beamforming with inter beam interference elimination technique compared to resource allocation beamforming technique without Inter beam interference elimination technique because of suppressed channel time variation. 


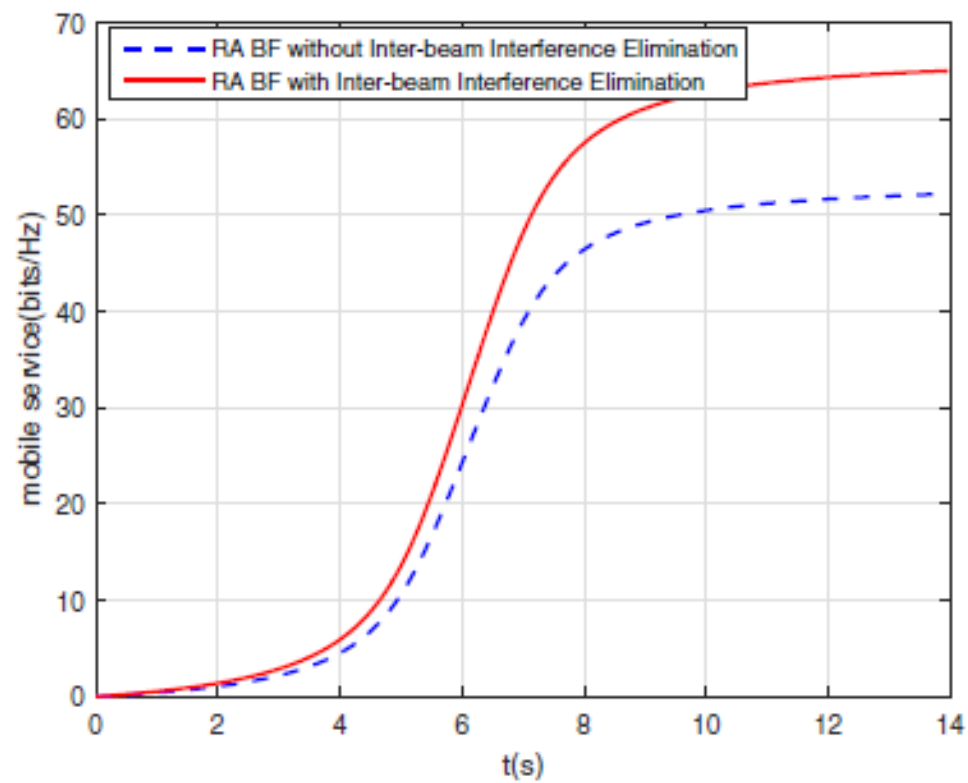

Figure 5. Mobile service comparison with different transmit power with/without inter beam interference elimination for resource allocation beamforming (RA BF) technique

\section{RESULT ANALYSIS}

There is always tradeoff between antenna beam gain and beam width. By increasing beam gain of antenna, beam width becomes narrow. Narrow beam width will decrease the probability of effective beamforming. There is optimum position aware beam adjustment technique in which switching interval of dominant radiating antenna is kept according to beamforming probability. By this technique antenna gain and beamwidth will be appropriate with good QoS.

To obtain multiple antenna gain, channel correlation must be weakened. Spatial modulation massive MIMO technique decreases ICI and thus channels correlation also reduces. This will ultimately increase multiple antenna gain. Spatial modulation activates only one transmit antenna at a time, which will reduce ICI.

Effectively Doppler spread is reduced by introducing common configurable amplitudes and phases (CCAP) parameters to optimize beamforming network. It will suppress residual Doppler shifts and will reduce channel time variations. Massive uniform linear array configured at transmitter provides multiple beamforming branch. Each beam will undergo only one dominant doppler frequency offset (DFO), which can be compensated at transmitter before transmitting the signal. Thus, channel time variation can be suppressed. Also, as number of Tx antenna increases Doppler spread decreases.

ICI reduction process in High mobility scenario for distributed antennas massive MIMO downlinks derived and total ICI matrix can be formulated as the weighting average of single ICI matrices. Where the weighting coefficients are the corresponding channel gain factors. By calculations unitary ICI matrixes derived and ICI reduction method without matrix inversion processes proposed with retaining same spectral efficiency.

\section{KEY FINDINGS AND FUTURE RESEARCH SCOPE}

High mobility of the wireless communication terminals faces numerous difficulties on the modelling, design, testing, analysis, and evaluations of 5G communication systems. Key challenges of high mobility communications are fast time-varying fading, channel estimation errors, doppler diversity, carrier frequency offset, inter carrier interference, high penetration loss and fast and frequent handovers. Key findings or opportunities for future research scope from in-depth literature survey can be pointed as:

- Channel estimation errors occur due to high mobility which seriously degrades system performance.

- Carrier frequency offset (CFO) is caused by mismatch between transmitter and receiver frequencies because of doppler shift.

- $\quad$ Doppler frequency offset (DFO) occur due to high mobility scenario.

- Inter beam interference introduces due to high mobility scenario.

- Channel time variation occurs due to dominant Doppler shift. 
- Inter carrier interference (ICI) is introduced by Doubly selective fading in high mobility systems in which channel changes inside single OFDM symbol and destroy the orthogonality among subcarriers

- In HSR systems, the well-sealed train carriages introduce severe penetration loss of the wireless signals.

- $\quad$ High mobility systems experiences frequent handovers.

Research gap found by literature review for performance analysis of massive MIMO for high speed railway is pointed as:

- Algorithm for elimination of inter beam Interference in beam forming of massive MIMO and analyze performance by changing number of Tx antennas for high speed train scenario.

- $\quad$ Reduce channel time variation via beam forming network optimization algorithm for massive MIMO downlink in high speed train.

- $\quad$ Receiver design for improving mean square error (MSE) and symbol error rate (SER) and performance analysis by increasing number of Rx antennas for high mobility scenario.

- Optimum pilot design for channel estimation in high mobility scenario for different environmental scenario like viaduct, tunnel, and urban.

- How to balance the tradeoff between the pilot overhead and the estimation performance in nonstationary high mobility systems also analysis for perfect CSI and imperfect CSI.

- Optimize number of antennas for beamforming network in massive MIMO to achieve lower doppler spread analyze with different modulation scheme in HSR.

- Low complexity Location aided beamforming solution to maximize the beam directivity for high mobility scenario.

- $\quad$ Low complexity ICI reduction methods for Rayleigh fading channel.

\section{CONCLUSION}

This article has reviewed recent advancements in technologies for high speed internet services for the travellers of high speed train. Initially significance of massive MIMO technology, which is the key role player of $5 \mathrm{G}$ technology is analysed for high mobility scenario. In most of the developed countries, high speed trains running at speed of around $500 \mathrm{~km} / \mathrm{h}$ are deployed but due to limitations of current wireless communication technology, high speed broadband services onboard the train is difficult to provide. For various essential services related to HD video streaming, video surveillance, internet of things, security and safety of public, high speed internet service with data rate of around $100 \mathrm{Mbps}$ is extremely necessary. In this article, various limitations or challenges to provide high speed internet on high speed train are analysed. High channel fading, high penetration loss, time varying nature of channel, channel estimation errors, doppler frequency offset, carrier frequency offset, Inter carrier interference and frequent handovers are some of the major challenges of wireless communication channel in high mobility scenario. To overcome such challenges intensive research work is going on across the globe. Some new revolutionary $5 \mathrm{G}$ transmission technologies like massive MIMO, mmWave technology, location aware beamforming, coordinated multi point cell structure are competent to minimize the losses and improve the wireless channel efficiency by giving higher throughput. In this article various current high speed train wireless channel models have been presented based on modelling approach and frequency range. Some techniques to address various challenges of high speed train are analysed with massive MIMO and other advanced technology. Also simulation results are plotted to analyse the performance of massive MIMO and beam forming techniques with various parameters like BER, doppler spread, number of antennas, SNR, and achievable channel capacity. In last, key findings from above analysis and based on that future research scope is presented.

\section{REFERENCES}

[1] T. L. Marzetta, "Massive MIMO: An Introduction," Bell labs technical journal, vol. 20, pp. 11-22, 2015, doi: 10.15325/BLTJ.2015.2407793.

[2] Y. Liu, C.-X. Wang, and J. Huang, "Recent Developments and Future Challenges in Channel Measurements and Models for 5G and Beyond High-Speed Train Communication Systems," IEEE Communications Magazine, vol. 57, no. 9, pp. 50-56, 2019, doi: 10.1109/MCOM.001.1800987.

[3] E. G. Larsson, O. Edfors, F. Tufvesson, T. L. Marzetta, "Massive MIMO for Next Generation Wireless Systems," IEEE Communications Magazine, vol. 52, no. 2, pp. 186-195, 2014, doi: 10.1109/MCOM.2014.6736761.

[4] Y. Liu, C.-X. Wang, J. Huang, J. Sun, and W. Zhang, "Novel 3D Non-Stationary MmWave Massive MIMO Channel Models for 5G High-Speed Train Wireless Communications," IEEE Transactions on Vehicular Technology, vol. 68, no. 3, pp. 2077-2086, 2019, doi: 10.1109/TVT.2018.2866414.

[5] A. G.-Plaza et al. "5G Communications in High Speed and Metropolitan Railways," 2017 11th European Conference on Antennas and Propagation (EUCAP), 2017, pp. 658-660, doi: 10.23919/EuCAP.2017.7928756. 
[6] J. Wu and P. Fan, "A survey on high mobility wireless communications: Challenges, opportunities and solutions," IEEE Access, vol. 4, pp. 450-476, 2016, doi: 10.1109/ACCESS.2016.2518085.

[7] D. Fan, Z. Zhong, G. Wang, and F. Gao, "Doppler Shift Estimation for High-Speed Railway Wireless Communication Systems with Large-Scale Linear Antennas," 2015 International Workshop on High Mobility Wireless Communications (HMWC), 2015, pp. 96-100, doi: 10.1109/HMWC.2015.7354343.

[8] B. Ai et al., "Challenges Toward Wireless Communications for High-Speed Railway," in IEEE Transactions on Intelligent Transportation Systems, vol. 15, no. 5, pp. 2143-2158, Oct. 2014, doi: 10.1109/TITS.2014.2310771.

[9] T. Li, X. Wang, P. Fan, and T. Riihonen, "Position-aided Large-scale MIMO Channel Estimation for High-Speed Railway Communication Systems," IEEE Transactions on Vehicular Technology, vol. 66, no. 10, pp. 8964-8978, 2017, doi: 10.1109/TVT.2017.2703595.

[10] X. Xiao, W. Muqing, Z. Ruojun, X. Jiaqi, and S. Xin, "Research on High-Speed Railway Model for Train-Ground MIMO Channel," 2014 International Symposium on Wireless Personal Multimedia Communications (WPMC), 2014, pp. 724-728, doi: 10.1109/WPMC.2014.7014910.

[11] Y. Sun, J. Wang, L. He, and J. Song, "Spectral Efficiency Analysis for Spatial Modulation in Massive MIMO Uplink over Dispersive Channels," 2017 IEEE International Conference on Communications (ICC), 2017, pp. 1-6, doi: 10.1109/ICC.2017.7996477.

[12] T. Zhou, C. Tao, and L. Liu, "LTE-assisted multi-link MIMO channel characterization for high-speed train communication systems," IEEE Transactions on Vehicular Technology, vol. 68, no. 3, pp. 2044-2051, 2019, doi: 10.1109/TVT.2018.2875526.

[13] Y. Qiu, H. Liu, and P. Fan, "Capacity Enhancement Using Cooperative Distributed Antenna System under LOS MIMO Channels in High Speed Train Scenario," The Sixth International Workshop on Signal Design and Its Applications in Communications, 2014, pp. 103-106, doi: 10.1109/IWSDA.2013.6849073.

[14] B. Hui, J. Kim, H.-S. Chung, Il-G. Kim, and H. Lee, "Efficient Doppler Mitigation for High Speed Rail Communication," 2016 18th International Conference on Advanced Communication Technology (ICACT), 2016, pp. 634-638, doi: 10.1109/ICACT.2016.7423500.

[15] X. Gao, O. Edfors, F. Rusek, and F. Tufvesson, "Massive MIMO Performance Evaluation Based on Measured Propagation Data," IEEE transactions on wireless communications, vol. 14, no. 7, pp. 3899-3911, 2015, doi: 10.1109/TWC.2015.2414413.

[16] C. Liao, K. Xu, W. Xie, and X. Xia, "3D massive MIMO channel model for high-speed railway wireless communication," Radio Science, vol. 55, no. 8, pp. 1-19, 2020, Art. no. e2020RS007070, doi: 10.1029/2020RS007070.

[17] T. Zhou, H. Li, Y. Wang, L. Liu, and C. Tao, "Channel modeling for future high speed railway communication systems: A Survey," IEEE Access, vol. 7, pp. 528180-52826, 2019, doi 10.1109/ACCESS.2019.2912408.

[18] J. Cao, R. Jiang, Y. Xu, and D. Li, "Position-aware Uplink Beamwidth Adjustment Scheme of Massive MIMO for High- Speed Railway Communications," 2019 IEEE 11th International Conference on Communication Software and Networks (ICCSN), 2019, pp. 303-308, doi: 10.1109/ICCSN.2019.8905319.

[19] D. Chizhik, "Slowing the Time-Fluctuating MIMO Channel by Beam Forming," IEEE Transactions On Wireless Communications, vol. 3, no. 5, pp. 1554-1565, 2004, doi: 10.1109/TWC.2004.833415.

[20] Y. Cui and X. Fang, "Performance Analysis of Massive Spatial Modulation MIMO in High Speed Railway," IEEE Transactions on Vehicular Technology, vol. 65, no. 11, pp. 8925-8932, 2016, doi: 10.1109/TVT.2016.2518710

[21] Y. Ge, W. Zhang, F. Gao, S. Zhang, and X. Ma, "Beamforming Network Optimization for Reducing Channel Time Variation in High-Mobility Massive MIMO," IEEE Transactions on Communications, vol. 67, no. 10, pp. 6781-6795, 2019, doi 10.1109/TCOMM.2019.2926464.

[22] W. Guo, W. Zhang, P. Mu, F. Gao, and H. Lin, "High-Mobility Wideband Massive MIMO Communications: Doppler Compensation, Analysis and Scaling Law," IEEE Transactions on Wireless Communications, vol. 18, no. 6, pp. 3177-3191, 2019, doi: 10.1109/TWC.2019.2911508.

[23] Ji. Lu, X. Chen, S. Liu, and P. Fan, "Location-aware ICI Reduction in MIMO-OFDM Downlinks for High-speed Railway Communication Systems," IEEE Transactions on Vehicular Technology, vol. 67, no. 4, pp. 2958-2972, doi: 10.1109/TVT.2017.2702186.

[24] X. Chen, P. Fan, "Low-complexity Location-aware Multi-user Massive MIMO Beamforming for High Speed Train Communications," 2017 IEEE 85th Vehicular Technology Conference (VTC Spring), 2017, pp. 1-6, doi: 10.1109/VTCSpring.2017.8108622.

[25] Y. Ge, W. Zhang, F. Gao, and H. Minn, "Angle-Domain Approach for Parameter Estimation in High-Mobility OFDM with Fully/Partly Calibrated Massive ULA," IEEE Transactions on Wireless Communications, vol. 18, no. 1, pp. 591-607, doi: 10.1109/TWC.2018.2883104.

\section{BIOGRAPHIES OF AUTHORS}

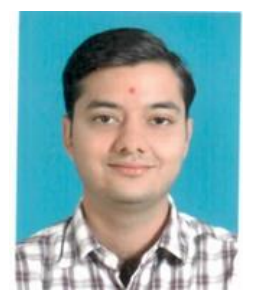

Maharshi K Bhatt, Assistant Professor Electronics and Communication Engineering Department, Government Engineering College, Bhuj, PIN code-370001, Gujarat, India. Research Scholar, Gujarat Technological University, Ahmedabad, Gujarat, India.

Email: maharshi88@gmail.com 


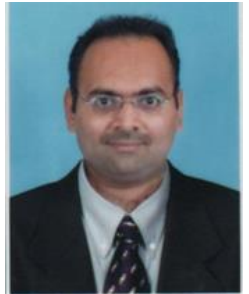

Bhavin S. Sedani, Professor, Electronics and Communication Engineering Department, L.D.College of Engineering, Ahmedabad, Gujarat, India. who has done his Ph.D. in Electronics and Communication Engineering with Wireless Communication field as field of specialization is working as Professor in L.D. College of Engineering Ahmedabad Gujarat. He has around 16 Year of experienceand Published and presented 45+ papers in various international journal and conferences.

Email: bhavin_s_sedani@yahoo.com

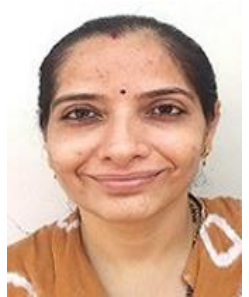

Komal R. Borisagar Associate Professor, Gujarat Technological University, Ahmedabad, Gujarat, India. Received B.E. degree in Electronics and Communication from C. U. Shah Engineering College, Saurashtra Universi ty, Rajkot, Gujarat, India in 2002 and M.E. degree in Communication System Engineering from Changa Institute of Technology, Gujarat University, and Ahmedabad in 2008. In 2012, she received her doctoral degree from the Department of Electronics and Communication Engineering, JJT Un iversity, Rajasthan. She has teaching experience of over 10 years. She has worked as Assistant Professor at Electronics and Communication Department, Atmiya Institute of Technology and Science, Rajkot, India. Currently she is working as Associate Professor, Gujarat Technological University, Ahmedabad, Gujarat, India.

Email: borisagar@gtu.edu.in 